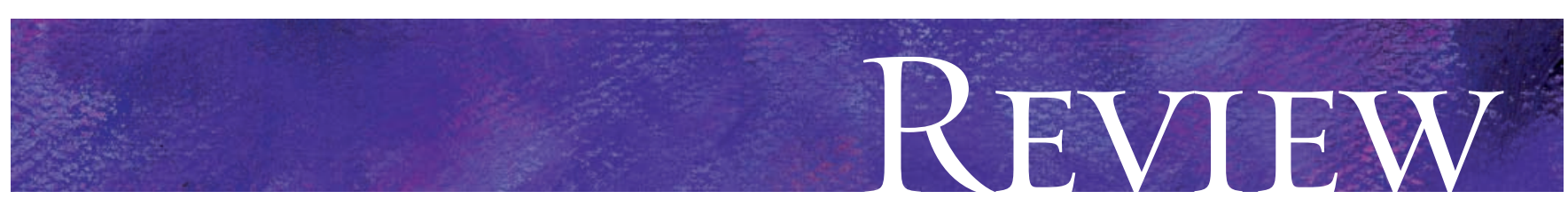

\title{
Clinical applications of cardiovascular magnetic resonance imaging
}

\author{
Constantin B. Marcu, Aernout M. Beek, Albert C. van Rossum
}

\section{ABSTRACT}

Cardiovascular magnetic resonance imaging (MRI) has evolved from an effective research tool into a clinically proven, safe and comprehensive imaging modality. It provides anatomic and functional information in acquired and congenital heart disease and is the most precise technique for quantification of ventricular volumes, function and mass. Owing to its excellent interstudy reproducibility, cardiovascular MRI is the optimal method for assessment of changes in ventricular parameters after therapeutic intervention. Delayed contrast enhancement is an accurate and robust method used in the diagnosis of ischemic and nonischemic cardiomyopathies and less common diseases, such as cardiac sarcoidosis and myocarditis. First-pass magnetic contrast myocardial perfusion is becoming an alternative to radionuclide techniques for the detection of coronary atherosclerotic disease. In this review we outline the techniques used in cardiovascular MRI and discuss the most common clinical applications.

CMAJ 2006;175(8):9II-7

$\mathrm{D}$ uring cardiovascular MRI, a patient is placed in the high-strength magnetic field of a superconducting magnet. Because heart movement with the cardiac cycle or respiration has a major impact on image quality, the electrocardiogram (ECG) is used to synchronize image acquisition with the cardiac-cycle phases (gating), and images are usually obtained during io to 20 seconds of breath-holding. Special sequences are applied to make the blood appear darker or brighter than the myocardium, generating static ("dark-blood" or "bright-blood") or dynamic (cine-"bright-blood") images.

Hydrogen nuclei (protons) behave like minuscule spinning magnets that have an alignment (spin, magnetic moment) parallel to the direction of the external magnetic field and a rotation (precession) frequency proportional to the strength of the field. ${ }^{1,2}$ When a radiowave with a frequency identical to the precession frequency is applied by a coil over a body region, the magnetic moment in that region will be flipped out at an angle to the magnetic field (excitation). The magnetic moment now has 2 components: one aligned with the external field (longitudinal magnetization) and the other perpendicular to the field (transverse magnetization). After excitation, the magnetic moment gradually returns to its baseline state (relaxation), the longitudinal magnetization increasing with a speed defined by the Ti-relaxation time and the transverse magnetization decreasing with a speed defined by the T2-relaxation time. Radio signals emitted during relaxation are detected by receiver coils and used to create the final image. ${ }^{1,2}$ The signal arising from different tissues is influenced by the Tr-longitudinal and $\mathrm{T} 2$-transverse relaxation times (which are tissue-specific and dependent on magnetic field strength), proton density, blood flow and the use of magnetic contrast media. ${ }^{1,2}$ Cardiovascular MRI images can be acquired (weighted) to show the distribution of tissue relaxation times ( $\mathrm{T}$ I and $\mathrm{T}_{2}$ ) or proton density. Gadoliniumcontaining paramagnetic contrast media (Gd-PCM) produce a decrease in $\mathrm{TI}$ that is directly proportional to the local concentration of Gd-PCM, which generally translates into increased signal intensity (brightness) in the area of interest. Magnetic contrast media are used for tissue characterization, to study myocardial perfusion and viability, and for angiography. ${ }^{2}$ The velocity of blood flow through vessels or cardiac valves can be measured by a process called velocity mapping. The product of blood velocity and cross-sectional area over time allows calculation of cardiac output, valvular regurgitant fraction and shunt ratios. ${ }^{2}$

Cardiovascular MRI has evolved over the last decade into a valuable tool for the diagnosis and management of a wide spectrum of cardiovascular disorders (Box I).

\section{Applications}

\section{Ventricular function and mass}

Cardiovascular MRI is the "gold standard" for quantifying ventricular volumes, ejection fraction and myocardial mass. ${ }^{2}$ ECG-gated, breath-holding, cine-"bright-blood" sequences are used to obtain a "stack" of contiguous, leftventricular (LV) short-axis slices 5-10 mm thick. ${ }^{3}$ Cardiovascular MRI is a tomographic technique that uses volumetric quantification based on Simpson's rule: the volume of a complex structure (ventricle) equals the sum of less complex subvolumes (short-axis slices). Endocardial and epicardial contours are drawn during post-processing ("off-line," after the MRI examination), generating enddiastolic, end-systolic and stroke volumes, ejection fraction (stroke volume divided by end-diastolic volume) and myo- 
Box 1: Indications for and contraindications to cardiovascular magnetic resonance imaging

\section{Current clinical applications}

- Assessment of left and right ventricular volumes and mass, as well as systolic function

- Assessment of myocardial viability

- Myocardial perfusion

- Evaluation of congenital heart disease with shunt calculation

- Evaluation and follow-up of valvular disease

- Evaluation of pericardial disease

- Evaluation of aortic disease

- Evaluation of cardiac masses

- Assessment of nonischemic cardiomyopathies

- Arrhythmogenic right ventricular cardiomyopathy

- Dilated cardiomyopathy

- Hypertrophic cardiomyopathy

- Myocarditis, sarcoidosis

Emerging applications

- Coronary angiography

- Interventional magnetic resonance

Contraindications and limitations

- Pacemakers and implanted cardioverter-defibrillators

- Neurostimulators, cochlear implants, vascular clips

- Claustrophobia

- Pregnancy (depending on risk:benefit ratio)

- Endocarditis

cardial mass (myocardial volume multiplied by musclespecific weight). In contrast, planar imaging modalities (2dimensional echocardiography and ventriculography) measure a limited number of surface areas and compare an object (ventricle) with a geometric model. Cardiovascular MRI yields more accurate values for LV parameters than planar imaging methods do, especially when the ventricular shape deviates from the assumed geometric model, as in ischemic or dilated cardiomyopathy, ${ }^{4-6}$ and is the most reliable way to assess regional and global right-ventricular (RV) function. Cardiovascular MRI is the best method for longitudinal follow-up of cardiovascular patients after therapeutic intervention owing to its excellent interstudy reproducibility, ${ }^{7-9}$ which also allows for reduced sample size, time and cost for clinical trials. ${ }^{7,10}$

\section{Myocardial viability}

During myocardial infarction (MI) a "wave" of cellular death moves from the endocardium toward the epicardium, which results in replacement of a variable amount of myocardium with scar. Impaired myocardial contraction in ischemic cardiomyopathy is not always a consequence of myocardial fibrosis alone. ${ }^{11}$ In some cases, there is myocardial "stunning": reduced contraction initially after MI despite successful reperfusion of the culprit artery, followed by spontaneous recovery within weeks. ${ }^{12}$ In other cases, there is myocardial "hibernation": impaired contraction in the absence of significant scar, a consequence of flow-limiting coronary stenosis with chronic ischemia. ${ }^{13}$

If myocardial contraction can be impaired by myocardial fibrosis, stunning or hibernation, how can these processes be differentiated, and will coronary revascularization procedures improve contraction? To answer these questions, we have to first define "myocardial viability." Clinically this term implies that, after absence of contraction during ischemia, contraction will be improved by coronary revascularization, ${ }^{14}$ whereas physiologically it means the absence of myocyte death.

In patients with ischemic cardiomyopathy the LV ejection fraction is a more powerful prognostic factor than the number of diseased coronary arteries. ${ }^{15-17}$ For patients with large amounts of jeopardized myocardium, the annual mortality is 4 times higher among those receiving medical treatment than among those with successful revascularization. ${ }^{18}$ Also, patients with a reduced LV ejection fraction and without much myocardial viability who undergo revascularization procedures have increased perioperative and long-term mortality rates. ${ }^{18}$ Thus, accurate discrimination between viable and nonviable myocardium is important in the decision about treatment strategy in ischemic cardiomyopathy.

With delayed contrast enhancement (DCE), a cardiovascular MRI technique that uses Gd-PCM, we can directly visualize areas of acute or chronic myocardial infarction or edema. The mechanism is hypothesized to be a combination of delayed wash-in/wash-out kinetics and different Gd-PCM volumes of distribution in regions of myocardial edema, necrosis or fibrosis compared with normal areas. ${ }^{2}$ The current DCE technique uses an inversion-recovery sequence for optimal contrast between infarcted (bright, "hyperenhanced") and normal (dark) myocardium. ${ }^{2,19-21}$ LV short-axis slices are acquired at the same positions as during cine-MRI, $5^{-20}$ minutes after injection of Gd-PCM..${ }^{19}$ Characteristically, an "ischemic" DCE pattern, invariably involving the subendocardial regions in the distribution territory of a coronary artery, is demonstrated in patients with MI. ${ }^{2,19,21}$ With its inplane spatial resolution of about $2 \times 2 \mathrm{~mm}$, DCE can differentiate subendocardial from subepicardial regions, allowing for the assessment of transmural infarct extension (Fig. I). Dysfunctional myocardial regions with less than $50 \%$ transmural infarct extension have a good chance of functional recovery after $\mathrm{MI}^{22}$ or with coronary artery bypass surgery. ${ }^{20,23}$ Even areas of myocardial thinning may recover after revascularization if there is less than $50 \%$ transmural scar (infarct) extension. ${ }^{24}$

Microvascular obstruction (blockage of capillaries by microemboli and endothelial edema) may be encountered in acute MI despite successful coronary revascularization. In such cases, a particular pattern of DCE is seen: dark subendocardial areas of microvascular obstruction, where contrast cannot penetrate, surrounded by a rim of hyperenhancement ("halo sign") (Fig. I, A and B).

DCE compares well with fluorodeoxyglucose positron emission tomography (FDG-PET), the "gold standard" for 
myocardial viability imaging. DCE has a sensitivity of $94 \%$ and a specificity of $84 \%$ when compared with FDG-PET in patients with ischemic cardiomyopathy and LV dysfunction. ${ }^{25}$ DCE performs as well as resting thallium-20I myocardial perfusion imaging with single photon emission computed tomography (SPECT) for detecting transmural infarctions (specificity $98 \%$ and $97 \%$, respectively) but is more accurate in detecting regions of subendocardial infarction (Fig. I, C and $\mathrm{D}$ ), which are missed by radionuclide techniques in $47 \%$ of myocardial segments and $13 \%$ of patients. ${ }^{26}$ Radionuclide techniques expose patients to a substantial amount of ionizing radiation, and PET is performed by relatively few specialized centres.

\section{Nonischemic cardiomyopathies}

\section{Dilated cardiomyopathy}

Having to differentiate between dilated and ischemic cardiomyopathy in patients with new-onset heart failure is common in clinical practice. Coronary angiography is often performed to provide a diagnosis, because patients with ischemic cardiomyopathy have a shorter survival time than patients with dilated cardiomyopathy ${ }^{27,28}$ and may benefit from coronary revascularization therapy. DCE may help clarify the cause. Myocardial scar with an "ischemic" pattern of DCE distribution is present in most patients with ischemic cardiomyopathy ${ }^{29,30}$ but in less than $15 \%$ of patients with dilated cardiomyopathy, ${ }^{30,31}$ which is consistent with findings from necropsy studies demonstrating visible scar in $\mathrm{I} 4 \%$ of cases. ${ }^{32}$ When found in patients thought to have dilated cardiomyopathy and without angiographic evidence of coronary disease, an "ischemic" DCE pattern is believed to result from prior, undiagnosed MI or coronary embolism. ${ }^{31} \mathrm{~A}$ "nonischemic" DCE pattern, characterized by patchy or linear midwall striae, has been described in $28 \%$ of patients with dilated cardiomyopathy. ${ }^{31}$ The extent of midwall enhancement is thought to have important negative prognostic implications for patients with dilated cardiomyopathy, independent of baseline ventricular parameters. ${ }^{33}$

\section{Hypertrophic cardiomyopathy}

Cardiovascular MRI can diagnose hypertrophic cardiomyopathy by demonstrating the distribution of hypertrophy and the functional consequences (dynamic outflow obstruction and mitral valve regurgitation). Compared with echocardiography, cardiovascular MRI can demonstrate more precisely the site and extent of hypertrophy, especially in the apical region. ${ }^{34}$ With DCE a patchy midwall distribution involving the hypertrophic regions is found in more than $80 \%$ of patients. ${ }^{35,36}$ The enhancement extent has been linked to the risk of sudden death, the presence of LV dilation and heart failure. ${ }^{36}$ Cardiovascular MRI can assess the effects of percutaneous septal ablation on the dynamic obstruction, mitral valve regurgitation and ventricular remodelling; DCE can accurately demonstrate the size and location of the iatrogenic septal infarction..$^{37,38}$

\section{Arrhythmogenic right-ventricular cardiomyopathy}

Arrhythmogenic RV cardiomyopathy is characterized by progressive fibro-fatty replacement involving variable regions of the right and left ventricles. Often the first manifestation is ventricular tachycardia or fibrillation in a young patient. Cardiovascular MRI is useful for diagnosing the condition when $\mathrm{RV}$ abnormalities, such as decreased global or regional wall motion or aneurysms, correspond to areas of fibro-fatty infiltration detected with TI-weighted imaging. ${ }^{2}$ DCE may demonstrate hyperenhancement in regions of fibro-fatty replacement or suggest an alternative cause (dilated cardiomyopathy or myocarditis) in young patients presenting with ventricular tachycardia. ${ }^{2}$ However, because arrhythmogenic RV cardiomyopathy can have a prolonged "silent" phase, a positive diagnosis should be based on established clinical criteria $^{39}$ in combination with imaging studies.

\section{Other cardiomyopathies}

Cardiac involvement, found in about $25 \%$ of patients with sarcoidosis on necropsy, is clinically apparent - as conduction abnormalities or ventricular tachycardia - in $5 \%$ of cases. ${ }^{2,40}$ Active sarcoidosis with myocardial inflammation appears as regions of bright signal on both $\mathrm{T} 2$-weighted sequences and with DCE..$^{40}$ Myocardial scar is visualized with DCE as bright midwall or transmural areas with a "noncoronary" distribu-

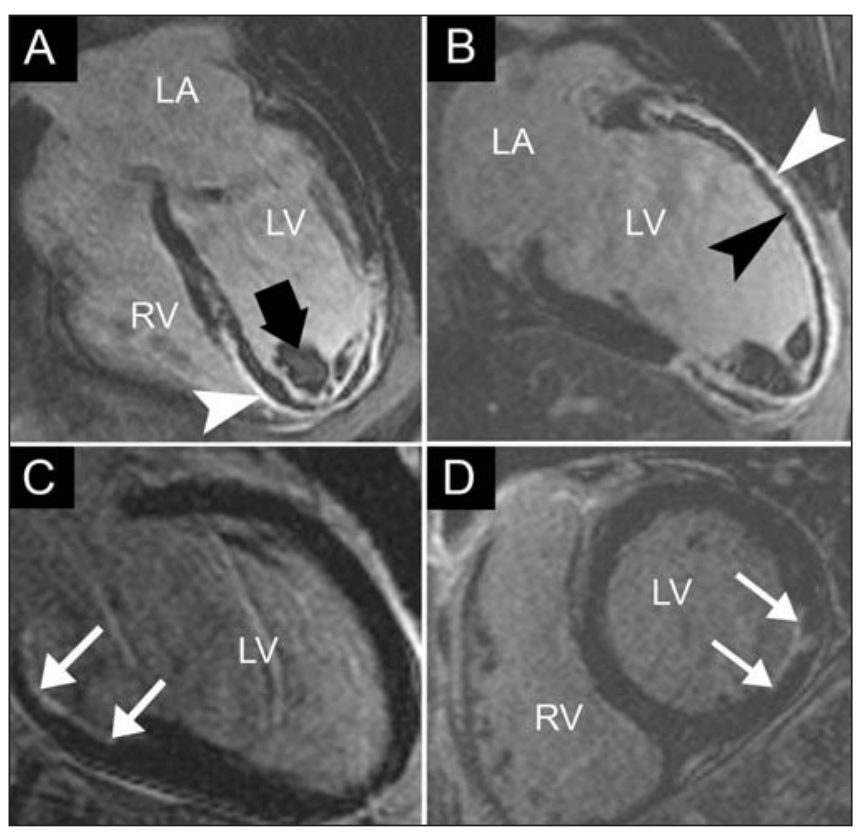

Fig. 1: Results of cardiovascular MRI with delayed contrast enhancement in myocardial infarction (MI). A and B: Four-chamber and 2-chamber views, respectively, of acute (48-hour) transmural anterior $\mathrm{Ml}$ with areas of microvascular obstruction (black arrowhead) surrounded by bright signal (white arrowhead), the "halo sign," and apical thrombus (black arrow). C and D: Twochamber and short-axis views, respectively, of old MI limited to the subendocardial region of the basal inferolateral wall (arrows). $L A=$ left atrium, $L V=$ left ventricle, $R V=$ right ventricle. 
tion (Fig. 2, A and B). DCE may be useful in assessing the effect of steroid therapy for cardiac sarcoidosis., ${ }^{2,40-43}$

In viral myocarditis cardiovascular MRI may demonstrate myocardial inflammation on T2-weighted images and with DCE during the acute phase or a "nonischemic" DCE pattern during the chronic phase (Fig. 2, C and D). ${ }^{2,40}$

\section{Pericardial disease}

Pericardial thickening or effusions are well defined by cardiovascular MRI. In constrictive pericarditis (Fig. 3, A and B) pericardial thickness can be measured and hemodynamic consequences (atrial dilation and diastolic interventricular septal bounce) evaluated. ${ }^{2,44}$ Real-time cine-MRI demonstrates interventricular dependence during respiration in pericardial tamponade or constriction. Pericardial calcification is not visualized with cardiovascular MRI; in such cases CT is an alternative. ${ }^{2}$

\section{Congenital heart disease}

Cardiovascular MRI is excellent for evaluating congenital heart disease, because its large field of view allows assessment of the anatomic relations between cardiac and vascular structures. In neonates and small children, echocardiography is the initial imaging modality of choice. Later on, especially
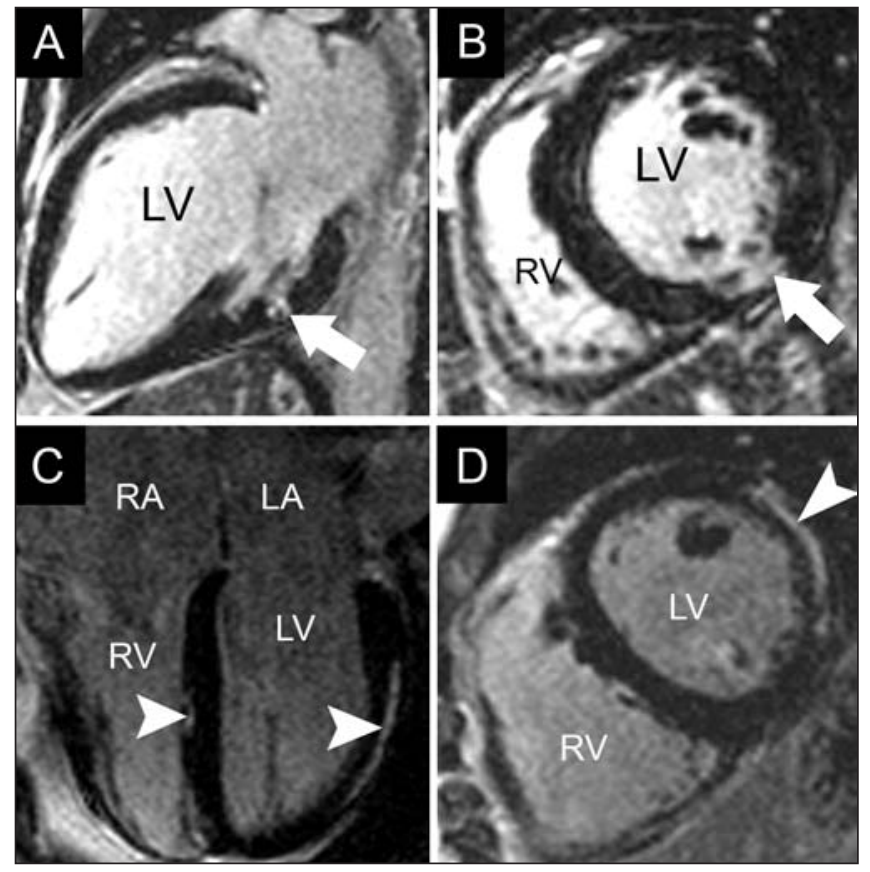

Fig. 2: Results of cardiovascular MRI with delayed contrast enhancement in sarcoidosis and myocarditis. A and B: Twochamber and short-axis views, respectively, of cardiac involvement in a patient with known pulmonary sarcoidosis, showing a small midwall area of myocardial hyperenhancement in the inferolateral region (arrow). C and D: Modified 4-chamber and short-axis views, respectively, of myocarditis with a subepicardial pattern of hyperenhancement in the lateral wall and a small region in the interventricular septum (arrowheads). RA = right atrium. after surgery, when scar tissue around the heart impedes ultrasound penetration and echocardiographic views are suboptimal, cardiovascular MRI is the preferred imaging method. ${ }^{2}$ Adolescents and adults with congenital heart disease often require serial examination, and cardiovascular MRI is preferred to cardiac catheterization or CT. ${ }^{2}$ MRI can identify a patent ductus arteriosus, an atrial or ventricular septal defect and anomalous pulmonary venous return, and shunt ratios can be calculated with the use of velocity mapping (Fig. 3, C and D).

\section{Disease of the aorta}

The structure of an aortic aneurysm and its relationship with branch vessels can be accurately defined by means of MRI. Cardiovascular MRI is useful in diagnosing the 3 variants of acute aortic syndrome: aortic dissection, intramural hematoma and penetrating aortic ulcer. ${ }^{2}$ In aortic dissection, MRI can demonstrate aortic valve regurgitation; Gd-PCM angiography is helpful in delineating the dissection flap and branchvessel involvement. MRI is useful in the follow-up after surgery for aortic dissection or aneurysm and in the diagnosis of aortic coarctation, and it is the method of choice for evaluating aortic involvement in Marfan's syndrome. ${ }^{2}$

\section{Valvular disease}

Cardiac MRI is complementary to echocardiography for assessment of valvular disease when echocardiographic images are of suboptimal quality, transesophageal echocardiography cannot be performed or the results of echocardiography and catheterization conflict. ${ }^{2}$ Small and very mobile valvular vegetations are not well visualized owing to insufficient temporal resolution; thus, MRI is not indicated for diagnosing endocarditis. CineMRI is used for evaluation of valve motion and direct measurement (planimetry) of valve area. ${ }^{2}$ Velocity mapping can quantify flow and regurgitation severity with incompetent valves and allow calculation of pressure gradients through stenotic valves by means of the modified Bernoulli equation: $\Delta \mathrm{P}(\mathrm{mm} \mathrm{Hg})=4 \times$ velocity $^{2}(\mathrm{~m} / \mathrm{s})$. Cardiovascular MRI is an excellent tool for follow-up of ventricular parameters in patients with valvular disorders ${ }^{2}$ and may be useful for defining the timing of valvular surgery. Prosthetic valves produce artifacts because of their metal content but can be imaged safely. ${ }^{45}$

\section{Myocardial perfusion and coronary artery imaging}

Myocardial-perfusion MRI allows visualization of the initial transit (first pass) of Gd-PCM through the heart. By means of ECG-gated, TI-weighted sequences with temporal resolution of at least every other heart beat, several short-axis LV slices can be imaged during I breath-holding. Passage of Gd-PCM appears as progression of a bright signal through the ventricular cavities and myocardium. Regions of decreased myocardial perfusion, a consequence of infarction or "ischemia" or both, will appear darker than normal regions. 
Several strategies are used for first-pass perfusion MRI in clinical practice. One strategy is to perform myocardial perfusion only during coronary vasodilation induced by adenosine or dypiridamole and then to use DCE to define myocardial viability. Another strategy involves acquiring images during both stress and rest. ${ }^{2}$ Stress/rest adenosine Gd-PCM firstpass myocardial perfusion with semiquantitative analysis has a sensitivity of $88 \%$, a specificity of $90 \%$ and an accuracy of $89 \%$ for detecting coronary artery disease in patients referred for coronary angiography. ${ }^{46}$ Semiquantitative analysis is timeconsuming and difficult in practice; therefore, many centres use visual interpretation for clinical studies. The diagnostic accuracy of visual interpretation with the use of a combination of stress/rest myocardial perfusion with MRI and DCE has recently been shown to be superior to stress/rest perfusion alone for the detection of significant coronary artery stenosis (accuracy of $88 \%$ v. $68 \%$ ) in patients with an intermediate pretest probability of coronary atherosclerosis referred for coronary angiography. ${ }^{47}$

The clinical value of myocardial perfusion with MRI for the detection of coronary artery disease is similar to that of radionuclide techniques, ${ }^{48,49}$ but MRI perfusion is performed in a single 45-minute session (compared with 2 sessions 3-24 hours apart for radionuclide techniques) and without ionizing radiation. However, less clinical-outcome information is available from MRI myocardial perfusion than from radionuclide techniques, which have been used for more than 30 years. A recent study of adenosine-stress perfusion MRI in patients with chest pain, negative troponin-I test results and nondiagnostic ECG findings demonstrated a sensitivity of I00\% and a specificity of $93 \%$ for the detection of future adverse cardiac outcomes. Patients with normal stress myocardial perfusion had no adverse cardiac outcomes during I year of follow-up. ${ }^{50}$

Coronary MRI angiography has been the subject of intense research over the past few years. Small vessel size and motion during the cardiac cycle make imaging the coronary arteries difficult. A multicentre study comparing 3-dimensional MRI and radiographic coronary angiography demonstrated negative predictive values for any coronary artery disease and for left main artery or 3-vessel disease of $81 \%$ and $100 \%$, respectively. ${ }^{51}$ However, in most cases, only the proximal segments of the coronary arteries can be visualized. This makes cardiovascular MRI useful for the noninvasive detection of anomalous origins of coronary arteries but of limited clinical value in patients with suspected coronary atherosclerotic disease.

\section{Cardiac masses}

Cardiovascular MRI is complementary to transthoracic echocardiography for the assessment of cardiac masses. Echocardiography may detect a suspicious cardiac structure but be unable to fully characterize it. With its larger field of view and unlimited imaging planes, MRI can more reliably demonstrate the location and extent of a mass. ${ }^{2}$ Although only pathological evaluation can establish a definite diagnosis, MRI offers opportunities for noninvasive tissue characterization with the use of TI/T2-weighted images. Malignant tu- mours and some benign masses, such as myxomas and hemangiomas, may demonstrate DCE; thrombi (Fig. I, A and B) do not enhance.

\section{Safety}

Cardiovascular MRI has no ionizing effects on tissues, and no long-term ill effects have been demonstrated. ${ }^{2}$ Metallic implants, such as hip and knee prostheses, modern cardiac valves, coronary stents and sternal wires, may produce local image artifacts (Fig. 3, A and B) but pose no hazard at field strengths of I.5 T and probably $3 \mathrm{~T}^{2,45}$ Patients with neurostimulators, cochlear implants, certain vascular clips and metal fragments retained in the eye must not undergo MRI or enter the scanner area. Pregnant women can undergo testing if the benefits of the procedure outweigh any theoretical risks to the embryo. ${ }^{52,53}$ The presence of a pacemaker or an implanted cardioverter-defibrillator contraindicates routine MRI. ${ }^{53}$ Exceptions for patients not dependent on the pacemaker should be considered on an individual basis. The patient has to be closely monitored by appropriately trained personnel during the examination, and the pacemaker has to be tested to confirm proper function after the study. ${ }^{53}$ The super-

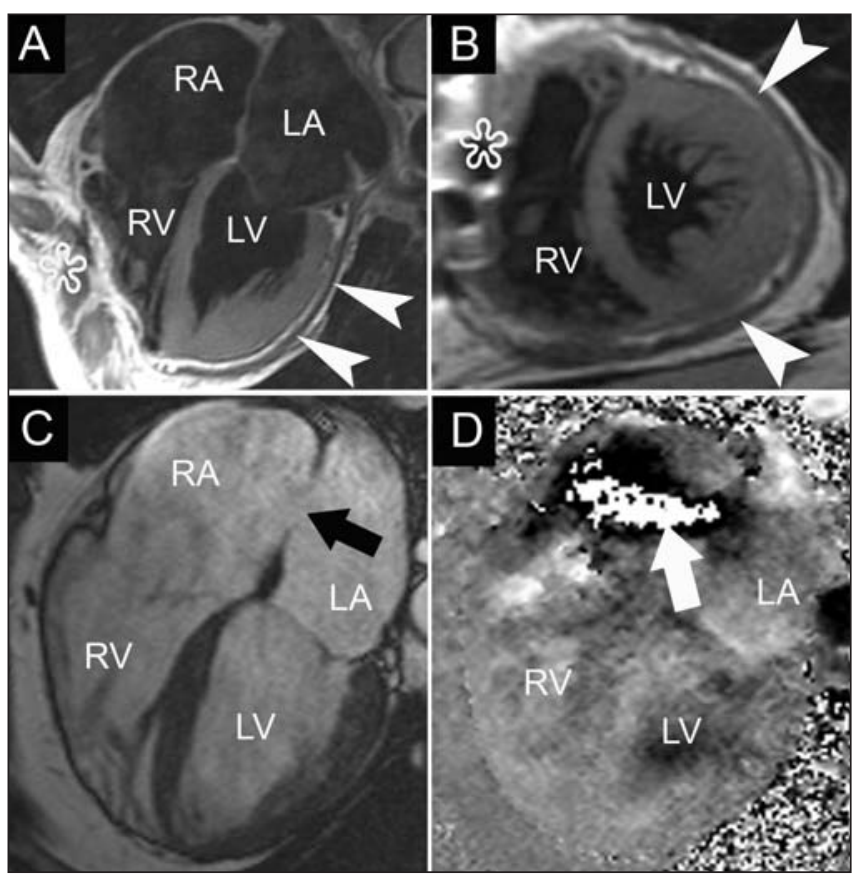

Fig. 3: Results of cardiovascular MRI in constrictive pericarditis and atrial septal defect. A and B: Four-chamber and short-axis T1-weighted ("dark-blood") breath-holding views, respectively, of constrictive pericarditis after coronary bypass surgery, showing thickened pericardium ( $7 \mathrm{~mm}$ ) (arrowheads), enlargement of both atria and a tubular right ventricle (RV). Asterisk indicates sternal wire artifacts. C and D: Four-chamber still frame from cine-MRI ("bright-blood") and 4-chamber velocity mapping image (in plane flow), respectively, of large atrial septal defect $(2 \times$ $2.5 \mathrm{~cm}$ ) (black arrow) with left-to-right shunt visible on the velocity-encoded image (white arrow) and enlarged right-sided chambers with a calculated ratio of pulmonary to systemic flow of 3.7 . 
conducting magnet is "always on"; therefore, ferromagnetic objects (oxygen tanks, wheelchairs and stretchers) should not be brought into the scanner room. ${ }^{2}$

Claustrophobia during MRI has been reported in $0.5 \%$ to $15 \%$ (average about $5 \%$ ) of patients. ${ }^{54,55}$ The use of benzodiazepines before the examination was shown to reduce the rate of unsuccessful examination due to claustrophobia from $4.4 \%$ to $1.6 \% .^{56}$

\section{Conclusion}

Cardiovascular MRI is a complex and dynamic technique that has entered the main stage of cardiovascular imaging. Its most important clinical applications are the evaluation of LV function in patients with suboptimal echocardiographic examinations, RV assessment, myocardial viability imaging, and follow-up of patients with congenital heart disease and diseases of the aorta.

Progress in scanner hardware and software will allow faster, more automated study, with a goal of whole-heart imaging during a single breath-holding. Advancements in MRI first-pass myocardial perfusion and coronary angiography may offer the possibility of "one-stop testing" for patients with coronary artery disease at an estimated cost of about US $\$ 600 .{ }^{2}$ The high cost for an initial comprehensive cardiovascular MRI test may be offset by cost reductions downstream related to decreased use of redundant imaging tests. ${ }^{2}$ With echocardiography as a reference, the estimated average cost for cardiovascular MRI is between that of cardiac CT or SPECT and that of FDG-PET or cardiac catheterization. ${ }^{2}$

Good communication between specialists in cardiovascular MRI and colleagues less familiar with the technology, during both patient referral (asking whether the test will help in the diagnosis of a particular condition) and reporting of the results (in a timely, well-structured and clear manner), is probably the most important factor for the appropriate clinical use of cardiovascular MRI.

\section{This article has been peer reviewed.}

From the Cardiac Diagnostic Unit, Hospital of Saint Raphael, New Haven, Conn. (Marcu), and the Department of Cardiology, VU University Medical Centre, Amsterdam, The Netherlands (Beek, van Rossum)

Competing interests: None declared.

Contributors: All of the authors drafted the article, revised it critically for important intellectual content and gave final approval of the version to be published.

\section{REFERENCES}

I. Hendrick RE. The AAPM/RSNA physics tutorial for residents. Basic physics of MR imaging: an introduction. Radiographics 1994;14:829-46.

2. Pennell DJ, Sechtem UP, Higgins CB, et al. Clinical indications for cardiovascular magnetic resonance (CMR): Consensus Panel report. Eur Heart J 2004;25:1940-65.

3. Barkhausen J, Ruehm SG, Goyen M, et al. MR evaluation of ventricular function: true fast imaging with steady-state free precession versus fast low-angle shot cine MR imaging: feasibility study. Radiology 2001;219:264-9.

4. Grothues F, Smith GC, Moon JC, et al. Comparison of interstudy reproducibility of cardiovascular magnetic resonance with two-dimensional echocardiography in normal subjects and in patients with heart failure or left ventricular hypertrophy. Am J Cardiol 2002;90:29-34.

5. Grothues F, Moon JC, Bellenger NG, et al. Interstudy reproducibility of right ven- tricular volumes, function, and mass with cardiovascular magnetic resonance. Am Heart J 2004; I47:218-23.

6. Bellenger NG, Burgess MI, Ray SG, et al. Comparison of left ventricular ejection fraction and volumes in heart failure by echocardiography, radionuclide ventriculography and cardiovascular magnetic resonance. Are they interchangeable? Eur Heart J 2000;21:1387-96.

7. Bellenger NG, Davies LC, Francis JM, et al. Reduction in sample size for studies of remodeling in heart failure by the use of cardiovascular magnetic resonance. J Cardiovasc Magn Reson 2000;2:27I-8.

8. Doherty NE III, Seelos KC, Suzuki J, et al. Application of cine nuclear magnetic resonance imaging for sequential evaluation of response to angiotensin-converting enzyme inhibitor therapy in dilated cardiomyopathy. J Am Coll Cardiol 1992;I9: 1294-302.

9. Bellenger NG, Marcus NJ, Davies C, et al. Left ventricular function and mass after orthotopic heart transplantation: a comparison of cardiovascular magnetic resonance with echocardiography. J Heart Lung Transplant 2000;19:444-52.

Io. Bellenger NG, Rajappan K, Rahman SL, et al. Effects of carvedilol on left ventricular remodeling in chronic stable heart failure: a cardiovascular magnetic resonance study. Heart 2004;90:760-4.

II. Lewis S, Sawada S, Ryan T, et al. Segmental wall motion abnormalities in the absence of clinically documented myocardial infarction: clinical significance and evidence of hibernating myocardium. Am Heart J I99I;I2I:I088-94.

I2. Heyndrickx GR, Millard RW, McRitchie RJ, et al. Regional myocardial functional and electrophysiological alterations after brief coronary artery occlusion in conscious dogs. J Clin Invest 1975;56:978-85.

I3. Rahimtoola SH. The hibernating myocardium. Am Heart JI989;II7:2II-2I.

I4. Gropler RJ, Bergman SR. Myocardial viability - What is the definition? J Nucl Med I99I;32:I0-2.

I5. Taylor G, Humphries J, Mellits E, et al. Predictors of clinical course, coronary anatomy and left ventricular function after recovery from acute myocardial infarction. Circulation i980;62:960-70.

I6. Mock MB, Ringqvist I, Fisher LD, et al. Survival of medically treated patients in the Coronary Artery Surgery Study (CASS) registry. Circulation I982;66:562-8.

I7. Sheehan FH. Determinants of improved left ventricular function after thrombolytic therapy in acute myocardial infarction. J Am Coll Cardiol I987;9:937-44.

I8. Allman KC, Shaw LJ, Hachamovitch R, et al. Myocardial viability testing and impact of revascularization on prognosis in patients with coronary artery disease and left ventricular dysfunction: a meta-analysis. J Am Coll Cardiol 2002;39:II5I-8.

19. Kim RJ, Shah DJ, Judd RM. How we perform delayed enhancement imaging. J Cardiovasc Magn Reson 2003;5:505-I4

20. Kim R, Wu E, Rafael A, et al. The use of contrast-enhanced magnetic resonance imaging to identify reversible myocardial dysfunction. N Engl J Med 2000;343: I $445-53$.

2I. Thomson LE, Kim RJ, Judd RM. Magnetic resonance imaging for the assessment of myocardial viability. J Magn Reson Imaging 2004;19:771-88.

22. Gerber BL, Garot J, Bluemke DA, et al. Accuracy of contrast-enhanced magnetic resonance imaging in predicting improvement of regional myocardial function in patients after acute myocardial infarction. Circulation 2002;106:1083-9.

23. Selvanayagam JB, Kardos A, Francis JM, et al. Value of delayed-enhancement cardiovascular magnetic resonance imaging in predicting myocardial viability after surgical revascularization. Circulation 2004;IIO:I535-4I.

24. Kim RJ, Shah DJ. Fundamental concepts in myocardial viability assessment revisited: when knowing how much is "alive" is not enough. Heart 2004;90:137-40.

25. Kuhl HP, Beek AM, van der Weerdt AP, et al. Myocardial viability in chronic ischemic heart disease: comparison of contrast-enhanced magnetic resonance imaging with (18)F-fluorodeoxyglucose positron emission tomography. J Am Coll Cardiol 2003;4I:I34I-8.

26. Wagner A, Mahrholdt H, Holly TA, et al. Contrast-enhanced MRI and routine single photon emission computed tomography (SPECT) perfusion imaging for detection of subendocardial myocardial infarcts: an imaging study. Lancet 2003;36I: 374-9.

27. Felker GM, Thompson RE, Hare JM. Underlying causes and long-term survival in patients with initially unexplained cardiomyopathy. N Engl J Med 2000;342:1077-84.

28. Felker GM, Shaw LK, O'Connor CM. A standardized definition of ischemic cardiomyopathy for use in clinical research. J Am Coll Cardiol 2002;39:210-8.

29. Wu E, Judd RM, Vargas J. Visualisation of presence, location, and transmural extent of healed Q-wave and non-Q-wave myocardial infarction. Lancet 200I;357:2I-8.

30. Bello D, Shah DJ, Farah GM. Gadolinium cardiovascular magnetic resonance predicts reversible myocardial dysfunction and remodeling in patients with heart failure undergoing beta-blocker therapy. Circulation 2003;108:1945-53.

3I. McCrohon JA, Moon JC, Prasad SK. Differentiation of heart failure related to dilated cardiomyopathy and coronary artery disease using gadolinium-enhanced cardiovascular magnetic resonance. Circulation 2003;108:54-9.

32. Roberts WC, Siegel RJ, McManus BM. Idiopathic dilated cardiomyopathy: analysis of 152 necropsy patients. Am J Cardiol I987;60:1340-55.

33. Assomull RG, Prasad SK, Burman E, et al. Cardiovascular magnetic resonance, fibrosis and prognosis in dilated cardiomyopathy [abstract]. J Cardiovasc Magn Reson 2006;8:3-4.

34. Moon JC, Fisher NG, McKenna WJ, et al. Detection of apical hypertrophic cardiomyopathy by cardiovascular magnetic resonance in patients with nondiagnostic echocardiography. Heart 2004;90:645-9.

35. Choudhury L, Mahrholdt H, Wagner A. Myocardial scarring in minimally symptomatic patients with hypertrophic cardiomyopathy. J Am Coll Cardiol 2002;40: $2156-64$. 
36. Moon JCC, McKenna WJ, McCrohon JA. Toward clinical risk assessment in hypertrophic cardiomyopathy with gadolinium cardiovascular magnetic resonance. J Am Coll Cardiol 2003;41:156I-7.

37. Van Dockum WG, ten Cate FJ, ten Berg JM, et al. Myocardial infarction after percutaneous transluminal septal myocardial ablation in hypertrophic obstructive cardiomyopathy: evaluation by contrast-enhanced magnetic resonance imaging. J Am Coll Cardiol 2004;43:27-34.

38. Van Dockum WG, Beek AM, ten Cate FJ, et al. Early onset and progression of left ventricular remodeling after alcohol septal ablation in hypertrophic obstructive cardiomyopathy. Circulation 2005;III:2503-8.

39. Corrado D, Fontaine G, Marcus FI, et al. Arrhythmogenic right ventricular dysplasia/cardiomyopathy: need for an international registry. Circulation 2000;I0I:EIoI-6.

40. Mahrholdt H, Wagner A, Judd RM, et al. Delayed enhancement cardiovascular magnetic resonance assessment of non-ischaemic cardiomyopathies. Eur Heart J 2005;26:I46I-74

4I. Shimada T, Shimada K, Sakane T, et al. Diagnosis of cardiac sarcoidosis and evaluation of the effects of steroid therapy by gadolinium-DTPA-enhanced magnetic resonance imaging. Am J Med 200I; IIO:520-7.

42. Vignaux O, Dhote R, Duboc D, et al. Clinical significance of myocardial magnetic resonance abnormalities in patients with sarcoidosis: a I-year follow-up study. Chest 2002;122:1895-90I.

43. Yazaki $Y$, Isobe $M$, Hiroe $M$, et al. Prognostic determinants of long-term survival in Japanese patients with cardiac sarcoidosis treated with prednisone. Am J Cardiol 200I;88:1006-10.

44. Masui T, Finck S, Higgins CB. Constrictive pericarditis and restrictive cardiomyopathy: evaluation with MR imaging. Radiology 1992;182:369-73.

45. Edwards MB, Taylor KM, Shellock FG. Prosthetic heart valves: evaluation of magnetic field interactions, heating, and artifacts at I.5 T. J Magn Reson Imaging 2000;12:363-9.

46. Nagel E, Klein C, Paetsch I, et al. Magnetic resonance perfusion measurements for the noninvasive detection of coronary artery disease. Circulation 2003;108:432-7.

47. Klem I, Heitner JF, Shah DJ, et al. Improved detection of coronary artery disease by stress perfusion cardiovascular magnetic resonance with the use of delayed enhancement infarction imaging. J Am Coll Cardiol 2006;47:1630-8.

48. Panting JR, Gatehouse PD, Yang GZ, et al. Echo-planar magnetic resonance myocardial perfusion imaging: parametric map analysis and comparison with thallium SPECT. JMagn Reson Imaging 2001;13:192-200.

49. Schwitter J, Nanz D, Kneifel S, et al. Assessment of myocardial perfusion in coronary artery disease by magnetic resonance: a comparison with positron emission tomography and coronary angiography. Circulation 2001;103:2230-5.

50. Ingkanisorn WP, Kwong RY, Bohme NS, et al. Prognosis of negative adenosine stress magnetic resonance in patients presenting to an emergency department with chest pain. JAm Coll Cardiol 2006;47:I427-32.

51. Kim WY, Danias PG, Stuber M, et al. Coronary magnetic resonance angiography for the detection of coronary stenoses. N Engl J Med 2001;345:1863-9.

52. Kanal E, Borgstede JP, Barkovich AJ, et al. American College of Radiology White Paper on MR Safety. AJR Am J Roentgenol 2002;178:1335-47.

53. Kanal E, Borgstede JP, Barkovitch AJ, et al. American College of Radiology White Paper on MR Safety: 2004 update and revisions. AJR Am J Roentgenol 2004; I82:IIII-4.

54. Sarji SA, Abdullah BJ, Kumar G, et al. Failed magnetic resonance imaging examinations due to claustrophobia. Australas Radiol r998;42:293-5.

55. Murphy KJ, Brunberg JA. Adult claustrophobia, anxiety and sedation in MRI. Magn Reson Imaging I997; $15: 5$ I-4.

56. Francis JM, Pennell DJ. Treatment of claustrophobia for cardiovascular magnetic resonance: use and effectiveness of mild sedation. J Cardiovasc Magn Reson 2000;2:139-4I

Correspondence to: Dr. Constantin B. Marcu, Cardiac Diagnostic Unit, Hospital of Saint Raphael, I45o Chapel St, New Haven CT o65II, USA; bogmarcu@pol.net

\section{Canadian Medical Association}

\section{Special Awards - Call for Nominations}

The Canadian Medical Association invites nominations for the 2007 special awards.

- Medal of Honour

- F.N.G. Starr Award

- Medal of Service

- May Cohen Award for Women Mentors

- Sir Charles Tupper Award for Political Action

- Award for Excellence in Health Promotion

- Award for Young Leaders

- Dr. William Marsden Award in Medical Ethics

Refer to the "Awards from CMA" section on cma.ca for detailed criteria on each of the awards or contact the awards co-ordinator at $800663-7336 \times 2280$

Nominations should be submitted to:

Chair, Committee on Archives and Awards c/o Committee Co-ordinator

Corporate Affairs

Canadian Medical Association

1867 Alta Vista Dr.

Ottawa ON K1G 3 Y6

Closing date for receipt of nominations is Nov. 30, 2006.

\section{Association médicale canadienne}

\section{Prix spéciaux pour l'an 2007 - Appel de candidatures}

L'Association médicale canadienne sollicite des candidatures à ses prix spéciaux pour l'an 2007.

- Médaille d'honneur

- Prix F.N.G. Starr

- Médaille de service

- Prix May-Cohen pour femmes mentors

- Prix Sir-Charles-Tupper d'action politique

- Prix d'excellence en promotion de la santé

- Prix des jeunes chefs de file

- Prix Dr-William-Marsden d'éthique médicale

Voir «Prix et distinctions de l'AMC» sur le site amc.ca pour les critères détaillés de chaque prix ou contacter la coordonnatrice des prix au $800663-7336$, poste 2280.

Les candidatures doivent être soumises à la :

Présidente, Comité des archives et des distinctions a/s Coordonnatrice des comités Affaires générale

Association médicale canadienne

1867, promenade Alta Vista

Ottawa (Ontario) K1G 3 Y6

Les candidatures doivent être présentées au plus tard le 30 novembre 2006. 